\title{
Dirac and Chiral Quantum Spin Liquids on the Honeycomb Lattice in a Magnetic Field
}

\author{
Zheng-Xin Liu ${ }^{1}$ and B. Normand ${ }^{2}$ \\ ${ }^{1}$ Department of Physics, Renmin University of China, Beijing 100872, China \\ ${ }^{2}$ Neutrons and Muons Research Division, Paul Scherrer Institute, CH-5232 Villigen-PSI, Switzerland
}

(Received 24 September 2017; revised manuscript received 11 December 2017; published 1 May 2018)

\begin{abstract}
Motivated by recent experimental observations in $\alpha-\mathrm{RuCl}_{3}$, we study the $K-\Gamma$ model on the honeycomb lattice in an external magnetic field. By a slave-particle representation and variational Monte Carlo calculations, we reproduce the phase transition from zigzag magnetic order to a field-induced disordered phase. The nature of this state depends crucially on the field orientation. For particular field directions in the honeycomb plane, we find a gapless Dirac spin liquid, in agreement with recent experiments on $\alpha-\mathrm{RuCl}_{3}$. For a range of out-of-plane fields, we predict the existence of a KalmeyerLaughlin-type chiral spin liquid, which would show an integer-quantized thermal Hall effect.
\end{abstract}

DOI: 10.1103/PhysRevLett.120.187201

The Kitaev model on the honeycomb lattice [1] is exactly solvable and thus presents a fundamental paradigm for both gapped and gapless quantum spin liquids (QSLs). An applied magnetic field can turn the gapless phase into a gapped, non-Abelian QSL, which would have direct applications in topological quantum computation [2]. Although Kitaev-type interactions are realized in layered honeycomb-lattice materials, such as $\mathrm{Na}_{2} \mathrm{IrO}_{3}$ [3-7] and $\alpha-\mathrm{RuCl}_{3}$ [8-10], their magnetically ordered ground states [6,7,11-14] preclude a QSL and indicate significant nonKitaev interactions $[4,15]$. Nevertheless, experimental observations of a continuum by inelastic neutron scattering (INS) [16] and a gapless mode at intermediate fields by thermal conductivity [17] have been taken as evidence for the proximity of $\alpha-\mathrm{RuCl}_{3}$ to Kitaev physics.

Recent experiments have established that magnetic order in $\alpha-\mathrm{RuCl}_{3}$ is weak and can be suppressed both by a magnetic field [17-19] and by pressure [20,21]. The critical field, when applied in the honeycomb $(a b)$ plane, is $B_{c}=7.5 \mathrm{~T}[17,19]$, which is far below the saturation field [22], and the resulting partially polarized but magnetically disordered state has been claimed to be a QSL. Numerous very recent studies of this phase are divided as to its nature, with nuclear magnetic resonance (NMR) in an out-of-plane field [18], specific heat [23,24], and neutron spectroscopy [25] reporting a gapped QSL, whereas power-law temperature dependences observed by thermal conductivity [17] and NMR in an in-plane field [19] suggest a gapless (nodal) QSL. NMR is a particularly sensitive probe of low-energy spin excitations and the spinlattice relaxation rate, $1 / T_{1} \propto T^{3}$, observed [19] at $T<10 \mathrm{~K}$ over a finite field range 7.5 $\mathrm{T}<B<12 \mathrm{~T}$ matches precisely the result anticipated for the point-node dispersion of a generic Kitaev system [26], albeit only at zero field. Because such nodal excitations are neither magnons nor the Majorana fermions of the Kitaev QSL, both of which show a gapped spin response in a field [27], their existence would pose a theoretical challenge. Because their density of states vanishes as energy approaches zero, their detection and distinction from a fully gapped phase poses a subtle experimental challenge.

In this Letter we analyze the low-energy physics of the field-induced magnetically disordered phase in $\alpha-\mathrm{RuCl}_{3}$. We employ a slave-particle representation to demonstrate that the properties of this state depend strongly on the direction of the applied field, which can produce a gapless Dirac QSL, a gapped chiral QSL, or a gapped and topologically trivial paramagnetic phase. By variational Monte Carlo (VMC) calculations using Gutzwillerprojected states, we obtain a semiquantitative description of the suppression of low-field order and deduce the dispersions and phase diagrams in all three cases.

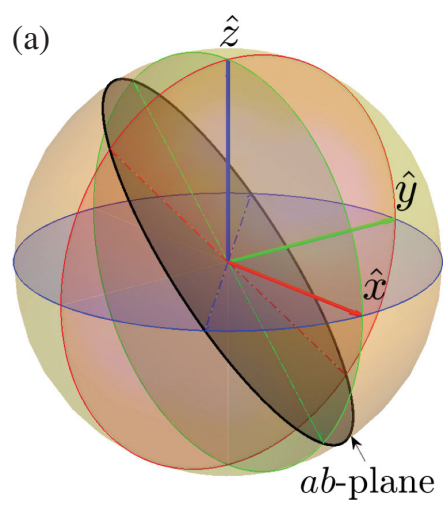

(b)

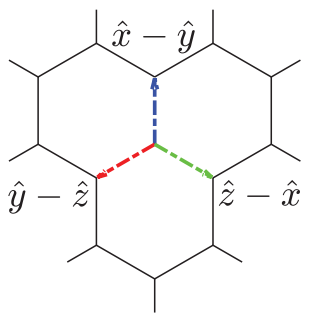

FIG. 1. (a) Field directions in the honeycomb system represented on the surface of a sphere. The dark plane is the lattice $a b$ plane. $\hat{x}, \hat{y}$, and $\hat{z}$ are the Kitaev axes of spins residing on the three different bonds [panel (b)]. Red, green, and blue planes are those normal to the respective spin axes. (b) Relation between lattice and spin basis vectors. 
We begin with a minimal effective model containing only Kitaev $(K)$ and symmetric off-diagonal $(\Gamma)$ terms,

$$
H=\sum_{\langle i, j\rangle \in \alpha \beta(\gamma)}\left[K S_{i}^{\gamma} S_{j}^{\gamma}+\Gamma\left(S_{i}^{\alpha} S_{j}^{\beta}+S_{i}^{\beta} S_{j}^{\alpha}\right)\right]+g \mu_{B} \sum_{i} \boldsymbol{B} \cdot \boldsymbol{S}_{i} .
$$

We neglect Heisenberg exchange terms, which are argued to be small in a perturbative expansion [28]. We adopt the representative parameters $K=-6.8 \mathrm{meV}$ and $\Gamma=$ $9.5 \mathrm{meV}$ used to fit the spin-wave spectrum of $\alpha-\mathrm{RuCl}_{3}$ measured by INS [29], which places the system far from the (generic) Kitaev regime [26]. Within this model we treat the Landé $g$ factor as isotropic $(g=2)$ [30].

Chemical coordination and spin-orbit coupling (SOC) in $\mathrm{Na}_{2} \mathrm{IrO}_{3}$ and $\alpha-\mathrm{RuCl}_{3}$ fix the spin axes of the Kitaev interaction terms to the lattice such that the crystalline $c$ axis is the [111] direction in the spin frame, as represented in Fig. 1(a). The lattice bond directions, which lie in the $a b$ plane and will turn out to have particular significance, are $(\hat{x}-\hat{y}),(\hat{y}-\hat{z})$, and $(\hat{z}-\hat{x})$ in the spin basis [Fig. 1(b)]. $\alpha-\mathrm{RuCl}_{3}$ has space-group symmetry $P 3_{1} 12$, whose point group is $D_{3}$. However, we restrict our considerations to a single honeycomb layer, which has point group $D_{3 d}$ when the applied field $\boldsymbol{B}=0$, and any lattice rotation is associated with a spin rotation due to SOC. A QSL state should break neither translation nor any of the symmetries remaining in the presence of $\boldsymbol{B}$.

Our analysis is based on a slave-particle representation in which spin operators at site $i$ are represented by two species of fermionic spinons, taking the quadratic forms $S_{i}^{m}=\frac{1}{2} C_{i}^{\dagger} \sigma_{m} C_{i}$, where $C_{i}^{\dagger}=\left(c_{i \uparrow}^{\dagger} \uparrow_{i \downarrow}^{\dagger}\right), m=x, y, z, \sigma_{m}$ are the Pauli matrices, and the spinons obey the on-site particle-number constraint $\hat{N}_{i}=c_{i \uparrow}^{\dagger} c_{i \uparrow}+c_{i \downarrow}^{\dagger} c_{i \downarrow}=1$. By applying the mean-field approximation detailed in Sec. S1 of the Supplemental Material (SM) [31], we express the $K-\Gamma$ model in the form

$$
\begin{aligned}
H_{\mathrm{mf}}= & \sum_{\langle i j\rangle \in \alpha \beta(\gamma)}\left[C_{i}^{\dagger}\left(t_{1}^{\gamma} R_{\alpha \beta}-i t_{0}^{\gamma}+t_{2}^{\gamma} \sigma_{\gamma}\right) C_{j}+\text { H.c. }\right] \\
& +g \mu_{B} \sum_{i} C_{i}^{\dagger}\left(\frac{1}{2} \boldsymbol{B} \cdot \boldsymbol{\sigma}+\lambda\right) C_{i}+H_{0},
\end{aligned}
$$

where $R_{\alpha \beta}=(-i / \sqrt{2})\left(\sigma_{\alpha}+\sigma_{\beta}\right), t_{1}^{\gamma}=-\frac{1}{2}|K|\left\langle C_{i}^{\dagger} R_{\alpha \beta} C_{j}\right\rangle^{*}$, $t_{0,2}^{\gamma}=-\frac{1}{8}(\Gamma-|K|)\left[\left\langle C_{i}^{\dagger} \sigma_{\alpha} R_{\alpha \beta} C_{j}\right\rangle^{*} \pm\left\langle C_{i}^{\dagger} \sigma_{\beta} R_{\alpha \beta} C_{j}\right\rangle^{*}\right], \lambda, \quad \mathrm{a}$ Lagrange multiplier corresponding to the average particle-number constraint, functions as a chemical potential, and $H_{0}$ is a constant. The $t_{1}^{\gamma}$ and $t_{2}^{\gamma}$ terms are analogous to the Rashba SOC of electrons [42]. We will determine all of these parameters by VMC calculations in which the local constraint is enforced exactly.

Before turning to this quantitative treatment, we consider the qualitative nature of the mean-field spinon state and its response to a magnetic field. The mean-field state is by construction a QSL, which in Eq. (2) has U(1) gauge symmetry because the spinon number is conserved. Finite spinon pairing terms may result in a $Z_{2}$ QSL [43], but are neglected in Eq. (2) because we have found (Sec. S1 of the SM [31]) that they are not favored energetically at intermediate magnetic fields.

The honeycomb lattice usually supports a conical spectrum due to its $C_{3 v}$ point-group symmetry. In graphene the $K$ and $K^{\prime}$ points are invariant under $C_{3 v}$, whose twodimensional irreducible representation results in twofold energetic degeneracies and hence in Dirac cones. These cones can be gapped in two ways, one being to add a sublattice chemical potential, $\mu_{z}$, which breaks the symmetry down to $C_{3}$. The alternative, which does not break the symmetry explicitly, is that increasing strain causes the two cones to move together adiabatically until they merge into a single branch, whose "semi-Dirac" [44] dispersion is quadratic in one $\boldsymbol{k}$-space direction but remains linear in the other, after which a full gap opens.

For illustration, we take $t_{1}^{\gamma}=1, t_{0}^{\gamma}=t_{2}^{\gamma}=0$ in Eq. (2). When $\boldsymbol{B}=0$, the spinon dispersion [Fig. 2(a)] is gapless with eight Dirac cones in the Brillouin zone [Fig. 2(b)]. These conical dispersions are protected by (emergent) symmetries, which separate them into two types.

(1) At $K$ and $K^{\prime}, C_{3 v}$ is preserved even with $\mathrm{SOC}$ and protects the cones. The generators of the $C_{3 v}$ group are

$$
\begin{aligned}
C_{3} & =e^{\mp i(2 \pi / 3)\left(\mu_{z} / 2\right)} \otimes e^{-i(2 \pi / 3)\left[\sigma_{c} /(2 \sqrt{3})\right]}, \\
M_{z} & =\mu_{ \pm} \otimes \frac{1}{\sqrt{2}}\left(\sigma_{x}-\sigma_{y}\right),
\end{aligned}
$$

at $K$ and $K^{\prime}$ respectively, where $\mu_{m}$ are Pauli matrices operating on the sublattice indices, $\mu_{ \pm}= \pm \frac{1}{2} \mu_{x}+$ $(\sqrt{3} / 2) \mu_{y}$, and $\sigma_{c}=\boldsymbol{\sigma} \cdot \hat{c}$ with $\hat{c}=(1 / \sqrt{3})(\hat{x}+\hat{y}+\hat{z})$. Like a $\mu_{z}$ term, a field (Zeeman) term, $H^{\prime}=B_{c} \sigma_{c}$, also breaks the mirror symmetry, gapping the cones at $K$ and $K^{\prime}$. Unlike $\mu_{z}$, which creates a trivial gapped phase, $B_{c}$ gives a nonzero Chern number, as detailed in Sec. S2 of the SM [31].

(2) At the $K_{x}$ and $K_{x}^{\prime}$ points, expansion of Eq. (2) in momentum space gives an effective Hamiltonian with $C_{4 v}$ symmetry, whose generators are represented as

$C_{4}=e^{\mp i \mu_{z}(\pi / 4)} \otimes e^{i \sigma_{x}(\pi / 4)}, \quad M_{x}=\mu_{x} \otimes \frac{1}{\sqrt{2}}\left(\sigma_{y}-\sigma_{z}\right)$,

at $K_{x}$ and $K_{x}^{\prime}$, respectively. Because the momentum itself is not invariant under $C_{4 v}$, this is an emergent symmetry, which is interpreted as operations with sublattice-spin coupling but without spatial rotation. As above, a magnetic field along $\hat{x}$ gaps the cones, with the key difference that the mass $B_{x}$ has the opposite sign to the mass $B_{c}$ for the cones at $K$ and $K^{\prime}$ [cf. Eqs. (3) and (4)], which has important consequences for the total Chern number. 


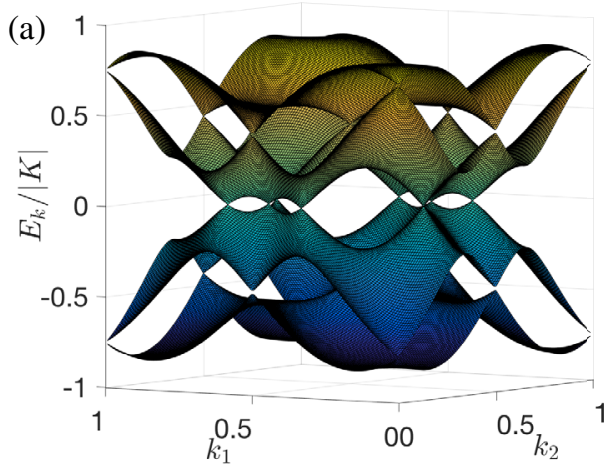

(b)

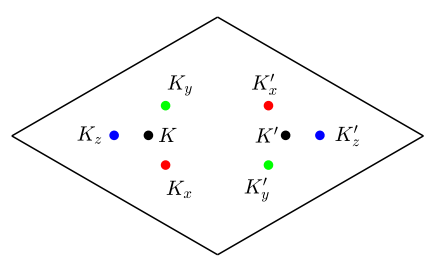

(c)

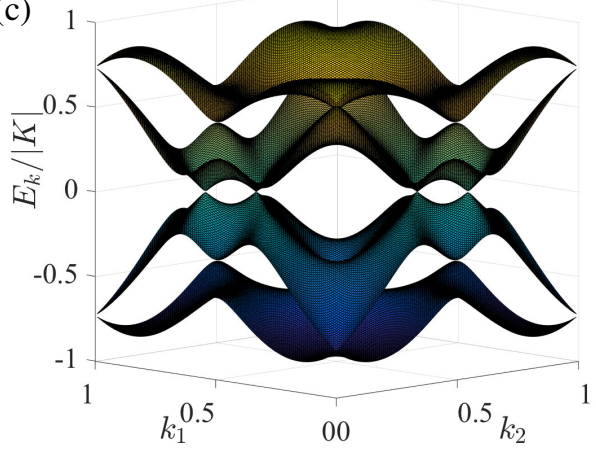

FIG. 2. (a) Spinon dispersion with $\boldsymbol{B}=0$, showing eight Dirac cones. (b) Locations of the eight nodes in the Brillouin zone. (c) Spinon dispersion with an in-plane field $\boldsymbol{B} \|(\hat{x}-\hat{y})$, showing two remaining pairs of Dirac cones close to $K, K^{\prime}$ and $K_{z}, K_{z}^{\prime}$.

The same physics applies to the Dirac cones at $K_{y}, K_{y}^{\prime}$ and $K_{z}, K_{z}^{\prime}$ in fields $B_{y}$ and $B_{z}$.

These results are summarized in Table I, where we have assumed that the Dirac cones are independent. Clearly if the magnetic field is oriented such that $\boldsymbol{B} \perp \hat{\alpha}$ and $\boldsymbol{B} \perp \hat{\beta}$ $(\alpha, \beta=c, x, y, z)$, i.e., at any intersection of the circles on the sphere in Fig. 1(a), then two pairs of Dirac cones have vanishing masses, as shown in Fig. 2(c). These cones are symmetry-protected and cannot be gapped individually. With increasing field, the cones of each pair move together, merging to a semi-Dirac dispersion, and then open a full gap, as shown in Sec. S3 of the SM [31].

For fields $\boldsymbol{B} \perp \hat{\alpha}$, i.e., any other points on the circles in Fig. 1(a), one pair of Dirac cones retains a vanishing linear mass and one may expect the spinons to remain gapless. However, higher-order processes generate a small gap that scales algebraically with the field, $\Delta=c|\boldsymbol{B}|^{a}$. We present some numerical results for $a$ in Sec. S3 of the SM [31]; as an example, $a=2.4$ if $\boldsymbol{B} \|(\hat{x}+\hat{y})$. The resulting slow growth of this gap may cause it to remain below the measurement temperature over a broad field range, making the system behave as if it were still gapless. This explains qualitatively the appearance of point-node excitations for all in-plane field directions in NMR [19].

For all other field directions, all the cones have nonzero masses and the spinon dispersion has a gap, which opens linearly in $|\boldsymbol{B}|$. The total Chern number of the half-filled fully gapped bands is $\mathcal{C}=\operatorname{sgn}(\boldsymbol{B} \cdot \hat{x})+\operatorname{sgn}(\boldsymbol{B} \cdot \hat{y})+$ $\operatorname{sgn}(\boldsymbol{B} \cdot \hat{z})-\operatorname{sgn}(\boldsymbol{B} \cdot \hat{c})$, which is either 0 or \pm 2 . If a point on the sphere of Fig. 1(a) is surrounded by only three arcs,

TABLE I. Action of magnetic fields in giving mass to the Dirac cones of Fig. 2(a).

\begin{tabular}{lccc}
\hline \hline Dirac node & Mass & Sign of mass & Chern number \\
\hline$K, K^{\prime}$ & $\boldsymbol{B} \cdot \hat{c}$ & - & $-\operatorname{sgn}(\boldsymbol{B} \cdot \hat{c})$ \\
$K_{x}, K_{x}^{\prime}$ & $\boldsymbol{B} \cdot \hat{x}$ & + & $\operatorname{sgn}(\boldsymbol{B} \cdot \hat{x})$ \\
$K_{y}, K_{y}^{\prime}$ & $\boldsymbol{B} \cdot \hat{y}$ & + & $\operatorname{sgn}(\boldsymbol{B} \cdot \hat{y})$ \\
$K_{z}, K_{z}^{\prime}$ & $\boldsymbol{B} \cdot \hat{z}$ & + & $\operatorname{sgn}(\boldsymbol{B} \cdot \hat{z})$ \\
\hline \hline
\end{tabular}

then $\mathcal{C}= \pm 2$ and a chiral QSL is obtained, which persists up to a critical value of $|\boldsymbol{B}|$ where its gap closes (Sec. S3 of the SM [31]). If a point is surrounded by four arcs, then $\mathcal{C}=0$ and the gap never closes at finite $|\boldsymbol{B}|$, meaning that the system is connected adiabatically to the fully polarized trivial (direct-product) state.

The topological transition between the $\mathcal{C}=0$ and \pm 2 regimes is a function of field angle and is discussed in Sec. S4 of the SM [31]. We recall that the field-induced QSL states we find have U(1) gauge symmetry, in contrast to the $Z_{2}$ gauge symmetry and finite vison gap of the Kitaev QSLs. In two spatial dimensions, U(1) gauge fields are confined, which is the trivial gapped phase, unless the matter (spinon) field is gapless, which is our four-cone case, or there is a Chern-Simons term, which is our $\mathcal{C}= \pm 2$ case. The transition may therefore be considered as a spinon (de)confinement process and its position obtained from the spinon dispersion and Chern number.

Thus at the mean-field level we have obtained crucial qualitative insight into the physical properties of three different field-induced disordered phases. However, the ground state observed in $\alpha-\mathrm{RuCl}_{3}$ is a zigzag magnetic order [11-14], which some authors [15] find in the classical $K-\Gamma$ model (1), whereas others [30] find that a small ferromagnetic (FM) Heisenberg interaction is required to stabilize it. In Sec. S1 of the SM [31] we discuss the quantum model and show that our results are reinforced by FM terms. To compare with experimental data, we introduce zigzag order within the single- $Q$ approximation, $\boldsymbol{M}_{i}=M\left(\sin \eta\left[\hat{e}_{i}^{x} \cos \left(\boldsymbol{Q} \cdot \boldsymbol{r}_{i}\right)+\hat{e}_{i}^{y} \sin \left(\boldsymbol{Q} \cdot \boldsymbol{r}_{i}\right)\right]+\cos \eta \hat{e}_{i}^{z}\right)$, where $\boldsymbol{Q}=[1 / 2,1 / 2]$, the local (spin) axes, $\hat{e}_{i}^{\alpha}$, are fixed by the classical ground state, and $\eta$ is the canting angle. We treat the static order as an external field to obtain a new mean-field Hamiltonian, $H_{\mathrm{mf}}^{\prime}=H_{\mathrm{mf}}-\sum_{i}\left(\frac{1}{2} \boldsymbol{M}_{i} \cdot C_{i}^{\dagger} \boldsymbol{\sigma} C_{i}+\right.$ H.c.); in a variational treatment, this process is equivalent to introducing an additional decoupling channel in $H_{\mathrm{mf}}$ (Sec. S1 of the SM [31]).

VMC calculations are based on the mean-field states but enforce the local constraint on spinon number by Gutzwiller projection. This method has been applied 


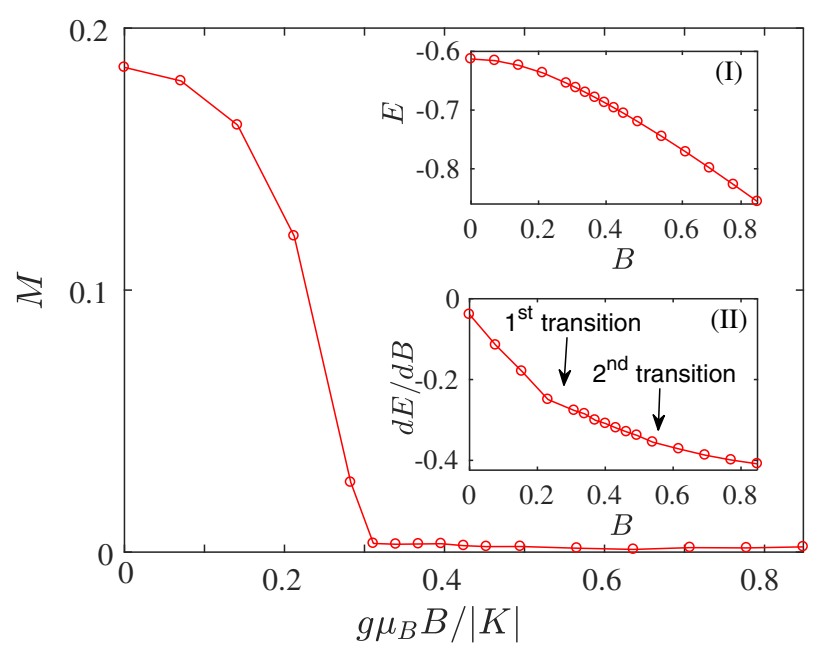

FIG. 3. Magnetization as a function of field for $\boldsymbol{B} \|(\hat{x}-\hat{y})$. Inset (I) shows the ground-state energy, $E$, as a function of $B$. Inset (II) shows $d E / d B$, whose discontinuity coincides with the magnetic transition. At the weak second transition, the Dirac cones become gapped.

widely to capture the essential physics of correlated electron systems and in certain cases, including slaveparton approaches to QSL states, can provide exact information. Here we employ the variational wave functions $\left|\psi_{G}(\boldsymbol{p})\right\rangle=P_{G}\left|\psi_{\mathrm{mf}}(\boldsymbol{p})\right\rangle$, where $\boldsymbol{p}$ denotes $\left(t_{0}^{\gamma}, t_{1}^{\gamma}, t_{2}^{\gamma}, \lambda\right.$, $M, \eta)$, to obtain the optimal state by minimizing the ground-state energy, $E=\left(\left\langle\psi_{G}|H| \psi_{G}\right\rangle /\left\langle\psi_{G} \mid \psi_{G}\right\rangle\right)$, on a system of 128 sites and use it to compute physical expectation values. To benchmark the accuracy of our results, in a small (8-site) system we obtain an overlap $\left\langle\psi_{\mathrm{VMC}} \mid \psi_{\mathrm{ED}}\right\rangle=0.988$ between the VMC and exactdiagonalization wave functions (more details are provided in Sec. S1 of the SM [31]).

We establish magnetic phase diagrams by fixing the field direction and increasing its magnitude. Zigzag order is suppressed for all field directions and vanishes at a lower critical field (Fig. 3), beyond which, as anticipated from the mean-field analysis, three different field-induced (partially polarized) disordered phases appear.

Dirac QSL.-If $\boldsymbol{B} \| \hat{\alpha}$ or $\boldsymbol{B} \|(\hat{\alpha}-\hat{\beta})(\alpha, \beta=x, y, z)$, there are two critical points, as shown in Fig. 4(a). The first marks the continuous transition from the ordered phase to a disordered one (Fig. 3) in which the spinon dispersion, with optimized parameters determined from VMC calculations, is gapless. Thus the intermediate phase is a stable U(1) Dirac QSL. At the second transition, which is also continuous, a gap opens as the system enters the trivial paramagnetic phase.

Chiral QSL.-If $\mathcal{C}= \pm 2$, for example when $\boldsymbol{B} \| \hat{c}$ or $\boldsymbol{B} \|\left(\hat{x}+\hat{y}+\frac{1}{2} \hat{z}\right)$ [Fig. 4(b)], the disordered phase is a chiral QSL $[45,46]$. This state is gapped, Abelian, has chiral edge modes, supports semionic spinon excitations, and has an integer-quantized thermal Hall conductivity, which can be (a)

(b)

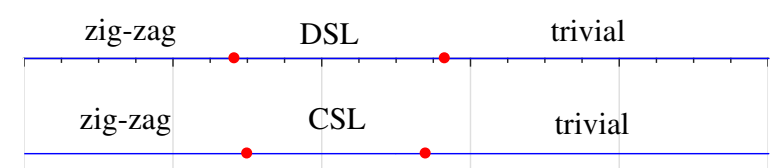

(c)

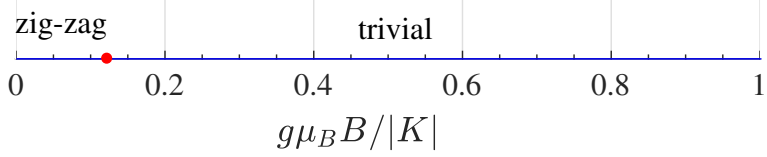

FIG. 4. Phase diagrams for different orientations of $\boldsymbol{B}$. (a) $\boldsymbol{B} \|(\hat{x}-\hat{y})$, where the intermediate phase is a field-induced Dirac spin liquid (DSL). Both transitions are of second order. (b) $\boldsymbol{B} \|\left(\hat{x}+\hat{y}+\frac{1}{2} \hat{z}\right)$, where the intermediate phase is a fieldinduced chiral spin liquid (CSL). The lower transition is of first order, while the upper is of second order. (c) $\boldsymbol{B} \|(\hat{x}+\hat{y}-2 \hat{z})$, where there is only one, weakly first-order, transition.

measured in experiment. It exists over a continuous regime of applied field directions, upon which the critical field depends strongly (numerically determined values include $g \mu_{B} B /|K| \simeq 2.4$ for $\boldsymbol{B} \| \hat{c}$ and 0.3 for $\boldsymbol{B} \|\left(\hat{x}+\hat{y}+\frac{1}{2} \hat{z}\right)$ [Fig. 4(b)]). At a higher critical point, the chiral QSL undergoes a transition to the trivial phase.

Gapped paramagnet.-For field orientations giving $\mathcal{C}=0$, and for the cases where the field direction lies on the circles in Fig. 1(a), the phase diagram has only one critical point [Fig. 4(c)]. This separates the ordered phase from the trivial polarized phase, whose gap opens linearly with field, except on the special lines where it opens algebraically with a higher power.

We have analyzed the spin-liquid state within a slaveparticle representation, which is uncontrolled. However, VMC studies allowing accurate enforcement of the constraint reveal only moderate quantitative alterations to the results. This indicates that the spinon description is well able to capture all the significant magnetic degrees of freedom. We stress that the gapless spin excitations of the field-induced U(1) QSL are deconfined Dirac fermions, which are different from the Majorana fermions in the gapless Kitaev QSL. Indeed, our results show that Kitaev QSLs are poor trial states, giving much higher energies than the U(1) QSL for the model of Eq. (1) with $\Gamma /|K|=1.4$. To address the stability of the U(1) QSL, we have tested the possibility of spinon pairing, but show in Sec. S1 of the SM [31] that this is not favorable. However, here we do not try to classify and test all possible QSL states of the model. We comment that recent numerical studies of the $K-\Gamma$ model $[47,48]$ also find QSL states over much of the phase diagram, albeit without detailed consideration of magnetic order or applied fields.

Our conclusions are in quantitative agreement with experiments on $\alpha-\mathrm{RuCl}_{3}$. The lower critical fields for the loss of zigzag order fall around 7.5 $\mathrm{T}$ for in-plane $(a b)$ fields. Our VMC phase diagrams in this case show that the field-induced disordered phase is either truly gapless for discrete field directions or otherwise has a very small gap 
that grows algebraically with $|\boldsymbol{B}|$, such that for temperatures $T \geq 1.5 \mathrm{~K}$ it would appear gapless. These results are fully consistent with recent NMR observations of a spin-lattice relaxation rate $1 / T_{1} \propto T^{3}$ and a largely isotropic response for all in-plane field orientations.

In summary, we have studied the $K-\Gamma$ model on the honeycomb lattice with an external magnetic field. By using Gutzwiller-projected states as variational wave functions and including zigzag magnetic order, we find three different field-induced disordered phases, whose nature varies strongly with the field direction. In certain cases, the intermediate QSL is gapless with Dirac-cone excitations, which are protected by emergent symmetries. In others it is a gapped chiral QSL, which may be sought in experiment through its integer-quantized thermal Hall effect.

We are grateful to $\mathrm{W}$. Yu and J. Wen for the experimental collaboration which initiated this study. We thank W. Ku, J.-X. Li, H.-J. Liao, C. Morais Smith, R. Valentí, M. Vojta, F. Wang, X.-Q. Wang, F. Wilczek, T. Xiang, J.-Z. Zhao, and Y. Zhou for helpful discussions. This work was supported by the National Natural Science Foundation of China (Grant No. 11574392), the Ministry of Science and Technology of China (Grant No. 2016YFA0300504), and the Fundamental Research Funds for the Central Universities and the Research Funds of Renmin University of China (No. 15XNLF19).

[1] A. Kitaev, Ann. Phys. (Amsterdam) 321, 2 (2006).

[2] C. Nayak, S. H. Simon, A. Stern, M. Freedman, and S. D. Sarma, Rev. Mod. Phys. 80, 1083 (2008).

[3] G. Jackeli and G. Khaliullin, Phys. Rev. Lett. 102, 017205 (2009).

[4] J. Chaloupka, G. Jackeli, and G. Khaliullin, Phys. Rev. Lett. 105, 027204 (2010).

[5] X. Liu, T. Berlijn, W.-G. Yin, W. Ku, A. Tsvelik, Y.-J. Kim, H. Gretarsson, Y. Singh, P. Gegenwart, and J. P. Hill, Phys. Rev. B 83, 220403 (2011).

[6] F. Ye, S. Chi, H. Cao, B. C. Chakoumakos, J. A. FernandezBaca, R. Custelcean, T. F. Qi, O. B. Korneta, and G. Cao, Phys. Rev. B 85, 180403 (2012).

[7] S. K. Choi, R. Coldea, A. N. Kolmogorov, T. Lancaster, I. I. Mazin, S. J. Blundell, P. G. Radaelli, Y. Singh, P. Gegenwart, K. R. Choi, S.-W. Cheong, P. J. Baker, C. Stock, and J. Taylor, Phys. Rev. Lett. 108, 127204 (2012).

[8] I. Pollini, Phys. Rev. B 53, 12769 (1996).

[9] K. W. Plumb, J. P. Clancy, L. J. Sandilands, V. V. Shankar, Y. F. Hu, K. S. Burch, H.-Y. Kee, and Y.-J. Kim, Phys. Rev. B 90, 041112 (2014).

[10] H.-S. Kim, V. Vijay Shankar, A. Catuneanu, and H.-Y. Kee, Phys. Rev. B 91, 241110 (2015).

[11] J. M. Fletcher, W. E. Gardner, A. C. Fox, and G. Topping, J. Chem. Soc. A 1038 (1967).

[12] J. A. Sears, M. Songvilay, K. W. Plumb, J. P. Clancy, Y. Qiu, Y. Zhao, D. Parshall, and Y.-J. Kim, Phys. Rev. B 91, 144420 (2015).
[13] R. D. Johnson, S. C. Williams, A. A. Haghighirad, J. Singleton, V. Zapf, P. Manuel, I. I. Mazin, Y. Li, H. O. Jeschke, R. Valentí, and R. Coldea, Phys. Rev. B 92, 235119 (2015).

[14] H. B. Cao, A. Banerjee, J.-Q. Yan, C. A. Bridges, M. D. Lumsden, D. Mandrus, D. A. Tennant, B. Chakoumakos, and S. E. Nagler, Phys. Rev. B 93, 134423 (2016).

[15] J. G. Rau, E.-H. Lee, and H.-Y. Kee, Phys. Rev. Lett. 112, 077204 (2014).

[16] A. Banerjee, C. A. Bridges, J. Q. Yan, A. A. Aczel, L. Li, M. B. Stone, G. E. Granroth, M. D. Lumsden, Y. Yiu, J. Knolle, S. Bhattacharjee, D. L. Kovrizhin, R. Moessner, D. A. Tennant, D. G. Mandrus, and S. E. Nagler, Nat. Mater. 15, 733 (2016).

[17] I. A. Leahy, C. A. Pocs, P. E. Siegfried, D. Graf, S.-H. Do, K.-Y. Choi, B. Normand, and M. Lee, Phys. Rev. Lett. 118, 187203 (2017).

[18] S.-H. Baek, S.-H. Do, K.-Y. Choi, Y. S. Kwon, A. U. B. Wolter, S. Nishimoto, J. van den Brink, and B. Büchner, Phys. Rev. Lett. 119, 037201 (2017).

[19] J. Zheng, K. Ran, T. Li, J. Wang, P. Wang, B. Liu, Z.-X. Liu, B. Normand, J. Wen, and W. Yu, Phys. Rev. Lett. 119, 227208 (2017).

[20] Z. Wang et al., arXiv:1705.06139.

[21] Y. Cui, J. Zheng, K. Ran, J. Wen, Z.-X. Liu, B. Liu, W. Guo, and W. Yu, Phys. Rev. B 96, 205147 (2017).

[22] R. D. Johnson, S. C. Williams, A. A. Haghighirad, J. Singleton, V. Zapf, P. Manuel, I. I. Mazin, Y. Li, H. O. Jeschke, R. Valentí, and R. Coldea, Phys. Rev. B 92, 235119 (2015).

[23] J. A. Sears, Y. Zhao, Z. Xu, J. W. Lynn, and Y.-J. Kim, Phys. Rev. B 95, 180411 (2017).

[24] A. U. B. Wolter, L. T. Corredor, L. Janssen, K. Nenkov, S. Schönecker, S.-H. Do, K.-Y. Choi, R. Albrecht, J. Hunger, T. Doert, M. Vojta, and B. Büchner, Phys. Rev. B 96, 041405 (2017).

[25] A. Banerjee, P. Lampen-Kelley, J. Knolle, C. Balz, A. A. Aczel, B. Winn, Y. Liu, D. Pajerowski, J.-Q. Yan, C. A. Bridges, A. T. Savici, B. C. Chakoumakos, M. D. Lumsden, D. A. Tennant, R. Moessner, D. G. Mandrus, and S. E. Nagler, npj Quantum Materials 3, 8 (2018).

[26] X.-Y. Song, Y.-Z. You, and L. Balents, Phys. Rev. Lett. 117, 037209 (2016).

[27] J. Knolle, D. L. Kovrizhin, J. T. Chalker, and R. Moessner, Phys. Rev. Lett. 112, 207203 (2014).

[28] W. Wang, Z.-Y. Dong, S.-L. Yu, and J.-X. Li, Phys. Rev. B 96, 115103 (2017).

[29] K. Ran, J. Wang, W. Wang, Z.-Y. Dong, X. Ren, S. Bao, S. Li, Z. Ma, Y. Gan, Y. Zhang, J. T. Park, G. Deng, S. Danilkin, S.-L. Yu, J.-X. Li, and J. Wen, Phys. Rev. Lett. 118, 107203 (2017).

[30] L. Janssen, E. C. Andrade, and M. Vojta, Phys. Rev. B 96, 064430 (2017).

[31] See Supplemental Material at http://link.aps.org/ supplemental/10.1103/PhysRevLett.120.187201, which contains Refs. [32-41], for details.

[32] I. Affleck, Z. Zou, T. Hsu, and P. W. Anderson, Phys. Rev. B 38, 745 (1988).

[33] N. Nagaosa, Quantum Field Theory in Strongly Correlated Electronic Systems (Springer, Heidelberg, 1999). 
[34] X.-G. Wen, Phys. Rev. B 65, 165113 (2002).

[35] Z.-X. Liu, Y. Zhou, H.-H. Tu, X.-G. Wen, and T.-K. Ng, Phys. Rev. B 85, 195144 (2012).

[36] S. M. Winter, Y. Li, H. O. Jeschke, and R. Valentí, Phys. Rev. B 93, 214431 (2016).

[37] S. M. Winter, K. Riedl, D. Kaib, R. Coldea, and R. Valentí, Phys. Rev. Lett. 120, 077203 (2018).

[38] R. Jackiw, Phys. Rev. D 29, 2375 (1984).

[39] F. D. M. Haldane, Phys. Rev. Lett. 61, 2015 (1988).

[40] H. Watanabe, Y. Hatsugai, and H. Aoki, Phys. Rev. B 82, 241403(R) (2010).

[41] M. Mohr, K. Papagelis, J. Maultzsch, and C. Thomsen, Phys. Rev. B 80, 205410 (2009).
[42] R. van Gelderen and C. M. Smith, Phys. Rev. B 81, 125435 (2010).

[43] Y.-Z. You, I. Kimchi, and A. Vishwanath, Phys. Rev. B 86, 085145 (2012).

[44] V. Pardo and W. E. Pickett, Phys. Rev. Lett. 102, 166803 (2009).

[45] V. Kalmeyer and R. B. Laughlin, Phys. Rev. Lett. 59, 2095 (1987).

[46] X. G. Wen, F. Wilczek, and A. Zee, Phys. Rev. B 39, 11413 (1989).

[47] A. Catuneanu, Y. Yamaji, G. Wachtel, H.-Y. Kee, and Y. B. Kim, arXiv:1701.07837.

[48] M. Gohlke, G. Wachtel, Y. Yamaji, F. Pollmann, and Y. B. Kim, Phys. Rev. B 97, 075126 (2018). 